\title{
Evaluación de las Competencias Docentes de Profesores Formados en Instituciones de Educación Superior: El Caso de la Asignatura de Tecnología en la Enseñanza Secundaria
}

\author{
Ricardo Morán, Edgar O. Cardoso*, , María T. Cerecedo y José C. Ortíz \\ Instituto Politécnico Nacional, Escuela Superior de Comercio y Administración (ESCA), Unidad Santo \\ Tomás, Sección de Estudios de Posgrado e Investigación, México, D. F., México (e-mail: \\ escastomoran@gmail.com, eoce@hotmail.com, tricermer@yahoo.com, carlos_acle@yahoo.com).
}

*Autor a quien debe ser dirigida la correspondencia

Recibido Oct. 24, 2014; Aceptado Dic. 1, 2014; Versión final recibida Ene. 2, 2015

\begin{abstract}
Resumen
El objetivo de esta investigación fue diagnosticar las competencias docentes en la asignatura de tecnología impartida en la enseñanza secundaria por profesores formados en Instituciones de Educación Superior en México. Como instrumento para recopilar la información se utilizó un cuestionario basado en una escala Likert y organizado por los factores de planeación, didáctica, evaluación y uso de tecnologías de la información y comunicación (TICs). La muestra total fue de 90 docentes distribuidos en secundarias generales y técnicas. Las principales conclusiones de la investigación fueron que los docentes poseen un nivel alto de dominio en las competencias relacionadas con la planeación, mientras que para los de didáctica, evaluación y manejo de TIC, los profesores tienen un dominio regular. Se recomienda a las Instituciones de Educación Superior en México establecer cursos de capacitación y de formación continua para estos docentes con la finalidad de brindarles estrategias que les permitan consolidar sus competencias académicas.
\end{abstract}

\section{Evaluation of teaching skills of Teachers Graduated from Institutes of Higher Education in México. The case of the subject technology in the Secondary School}

\begin{abstract}
The objective of this research was to assess teaching skills in the subject taught in secondary education technology by professors formed in Higher Education Institutions in México. A survey based on a Likert scale and organized by the factors of planning, teaching, evaluation and use of information and communication technologies (ICTs). The total sample included 90 teachers coming from general and technical secondary schools. The main conclusions of this research were that teachers have a high level of proficiency in skills related to planning, while for didactic, assessment and use of ICTs, teachers have an average domain. It is recommended that Higher Education Institutions should establish continuing education courses so these teachers showing some deficiencies could learn new strategies and can improve their academic competences.
\end{abstract}

Keywords: teaching skills, technology, assessment, teacher formation 


\section{INTRODUCCIÓN}

La tecnología se ha constituido como un elemento fundamental para el desarrollo integral de los estudiantes en el siglo XXI con una visión humanista caracterizada por ofrecer a los educandos los elementos para una formación tecnológica básica, que permita la comprensión y utilización de los procesos productivos, sus usos así como las consecuencias éticas, ambientales, sociales y económicas (Ramírez y Escalante, 2007). Así, la importancia de la tecnología es desarrollar herramientas para uso cotidiano por lo que se le define como el área de conocimiento que estudia a la técnica, sus funciones, los insumos y los medios que la conforman, sus procesos de cambio, así como su interacción con el contexto sociocultural y natural. En este sentido, la tecnología tiene una perspectiva local debido a que responde a las necesidades regionales de los países (Rodríguez, 1998). Según Martín y González (2002), en España e lberoamérica a partir de las reformas educativas de los años noventa, se generó un lugar en los currículos básicos en las etapas obligatorias para la enseñanza de la tecnología, específicamente a nivel secundaria, por lo que esta asignatura se le concedió un papel en la formación integral de los estudiantes. Específicamente, la relevancia de incluir en el plan de estudio de educación secundaria la asignatura de tecnología radica en promover el desarrollo de competencias genéricas y específicas relacionadas con los diversos instrumentos y medios técnicos que posibilitan la transformación de los insumos en bienes tangibles e intangibles que las sociedades requieren en ambientes específicos para satisfacer sus necesidades primordiales y así, estar en posibilidades de proveer un primer acercamiento al ámbito laboral para los estudiantes.

Para el caso de México, dicha asignatura se encuentra ubicada en el Estándar Curricular denominado "Exploración y comprensión del mundo natural y social". Tiene como antecedente inicial el nivel de preescolar con el campo formativo de desarrollo físico y salud; mientras que para los tres primeros grados de nivel primaria, se relaciona con "Exploración de la naturaleza y la sociedad" así como la "Entidad donde vivo"; en tanto que en los tres últimos grados de primaria, se vincula con Geografía e Historia por lo que al llegar a secundaria, se le ha establecido como Tecnología I, II y III (SEP, 2011). Asimismo, dicha materia comparte el mismo estándar curricular con Geografía de México y del Mundo I y II junto con la llamada Asignatura Estatal. En cuanto a su estructura curricular, la asignatura posee seis campos tecnológicos: tecnologías agropecuarias y pesqueras; tecnologías de los alimentos; tecnologías de la producción; tecnologías de la construcción; tecnologías de la información y comunicación y tecnologías de la salud, los servicios y la recreación (Diario Oficial de la Federación, 2011). La relevancia de estos campos es orientar a los profesores acerca de su trabajo porque definen los contenidos y sugerencias didácticas para favorecer los aprendizajes de los estudiantes. De esta manera, para el desarrollo de los procesos formativos de esta asignatura, la Secretaría de Educación Pública (SEP, 2009) concibe al docente como un agente relevante con un perfil distinto integrado por aspectos epistemológicos, prácticos relacionados con el saber hacer, habilidades pedagógico - didácticas y una postura actitudinal crítica sobre los valores que sustentan la educación tecnológica, lo cual tiene su fundamento principal en la formación universitaria inicial que tuvo el profesor para posteriormente consolidar su práctica docente a través de la experiencia.

Así, es necesario que el profesor posea un conjunto de múltiples saberes tecnológicos que abarcan desde ámbitos tales como la ciencia, tecnología y sociedad; diseño, proyecto tecnológico y análisis de producto; sistemas técnicos y sociotécnicos; sistemas de representación; sistemas productivos; tecnologías de gestión y sistemas ambientales, entre otros. También el docente de esta asignatura es relevante que domine los conocimientos disciplinarios, las habilidades técnicas y la didáctica propia de la asignatura como son los conocimientos sobre planeación, estrategias para la enseñanza y tipos e instrumentos de evaluación (Diario Oficial de la Federación, 2011). Para alcanzarlo, es relevante establecer que para lograr el perfil de egreso de la educación básica se utilice el enfoque por competencias. En este sentido, el concepto de competencias ha sido definido por diversos autores (Tobon, 2006; Zabala y Arnau, 2007 y Tejada, 2009), los cuales coinciden en que es un término holístico que incluye habilidades, destrezas y actitudes en un contexto específico en donde se utilizan no sólo en forma conceptual y procedimental sino también actitudinal. De ahí, que la experiencia del sujeto es un componente fundamental que le permite utilizar sus diversos saberes (conocer, hacer y ser) con la finalidad de proponer soluciones acordes a la situación 0 problema al que se enfrenta. Como lo menciona Perrenoud (2004), el ejercicio de las competencias pasa por operaciones mentales complejas, sustentadas por esquemas de pensamiento, que permiten determinar (de un modo consciente y rápido) y realizar (de un modo eficaz) una acción relativamente adaptada a la situación; por lo que las competencias profesionales se crean en formación, pero también a merced de la navegación cotidiana del practicante, de una situación de trabajo a otra.

Con base en Zabalza (2003), el cual establece las siguientes competencias docentes: Planificar el proceso enseñanza-aprendizaje; seleccionar y preparar los contenidos disciplinares; ofrecer informaciones y explicaciones comprensibles y bien organizadas; manejo de las nuevas tecnologías; diseñar metodologías y organizar actividades; comunicarse-relacionarse con los alumnos; tutorizar; evaluar; reflexionar e investigar sobre la enseñanza e identificarse con la institución y trabajar en equipo. Por su parte, Bozu y Canto (2009) establecen que para una docencia de calidad, los profesores tienen que poseer un conjunto de 
competencias profesionales generales y específicas: 1) Competencias comunicacionales; 2) Competencias organizativas; 3) Competencias de liderazgo pedagógico; 4) Competencias científicas y 5) Competencias de evaluación y control. En este sentido, Donoso (2008) y Poggi (2011) establecen que la calidad educativa está asociada a las competencias desarrolladas por los docentes en la sociedad del conocimiento, por lo que es necesario establecer estudios relacionados con la formación inicial y continua, así como evaluaciones que valoren el nivel de dominio de los profesores para los ámbitos de planeación, didáctica, evaluación, y manejo de las herramientas tecnológicas.

Por consiguiente, la relevancia de evaluar las competencias docentes radica en que son los responsables de implementar, organizar y evaluar los programas educativos de la institución en la que laboran. Así, este proceso comienza con una autoevaluación basada en criterios orientados hacia el desempeño global del profesorado con la finalidad de valorar el dominio alcanzado en las áreas relacionadas con la planificación, la tutoría, el enfoque metodológico, la tecnológica y la evaluativa con la finalidad de elaborar el diagnóstico sobre las fortalezas y debilidades de la comunidad académica para así establecer procesos de formación continua (Palominos, et al., 2014). De este modo, los estudios previos relacionados con esta temática de las competencias docentes se han enfocado a las de carácter académico (Cardoso, et al., 2013) en los cuales los principales resultados indican un nivel de consolidación medio en los docentes en cuanto a sus competencias relacionadas con sus estrategias didácticas, instrumentos de evaluación, así como su papel como tutores durante los procesos formativos de los estudiantes. Otro tipo de investigaciones se han enfocado a las competencias de tipo científico de los profesores (Valdés, et al., 2012; Vera, 2014 y Hodgson, et al., 2014,), hallando niveles regulares en aquellas competencias enfocadas a la asesoría de tesis, trabajo colaborativo entre el investigador con sus alumnos y la participación en la escritura de textos académicos. De la misma manera, hay estudios acerca del empleo de las TIC por parte de los docentes en donde se han encontrado que la mayoría se orientan a emplearlas en actividades básicas como la elaboración de pruebas o datos relacionados con su plan de clase, para la producción de materiales didácticos, así como para realizar investigaciones a partir de diversas fuentes (Rangel y Peñalosa, 2013; Gibson, 2013; Vera, et al., 2014).

En lo que respecta a los estudios previos sobre la asignatura de tecnología, se tienen los realizados por Martín y González (2002), Ferreras (2004) y Alcira, et al. (2008), Pantoja y Huertas (2010), los cuales se enfocaron hacia la relevancia de la educación tecnológica en los planes de estudio proponiendo estrategias de enseñanza (casos simulados, resolución de problemas técnicos, el método de proyectos y generación de comunidades de investigación), el enfoque didáctico basado en ciencia, tecnología y sociedad (CTS), así como la integración de las TIC en las actividades de docencia, en donde la mayoría de los profesores reconocieron su insuficiente formación en estas herramientas tecnológicas, así como que la preparación de los materiales informáticos para el aula es laboriosa y que el software no es el adecuado a los contenidos de la asignatura. De esta manera, es importante señalar que los profesores que imparten la asignatura de tecnología tuvieron su formación académica inicial en las instituciones de educación superior (IES), ya sea en las universidades o en los institutos tecnológicos tanto de régimen público o privado, las cuales les proporcionaron un conjunto de fundamentos teóricos y metodológicos sobre las competencias docentes para utilizarlas en su actividad académica. En este sentido, el estudio es un primer acercamiento hacia la enseñanza y formación universitaria brindada por las IES en México, lo cual sirve de diagnóstico para determinar si éstas proveen de las competencias idóneas para que los profesores de la asignatura de tecnología proporcionen un servicio de calidad a los estudiantes. Así, como lo menciona Palominos, et al. (2014), es relevante identificar si los modelos de formación universitaria han adoptado el enfoque de la educación basada en competencias como un proceso de renovación curricular que haya impactado en forma innovadora o si se han quedado a nivel de formulario dejando una visión fragmentada y sesgada.

Con base en lo anterior, es relevante establecer líneas de investigación relacionadas con los profesores que imparten la asignatura de Tecnología en lo que respecta a la organización de sus actividades en el aula, por lo que es relevante diagnosticar sus competencias docentes en los ámbitos de planeación, didáctica, evaluación y el uso de las TIC, para así estar en posibilidades de diseñar opciones de formación universitaria, ya sea a nivel de capacitación o posgrado.

\section{METODOLOGÍA}

Con fundamento en Namakforoosh (2011), el tipo de investigación que se empleó fue de tipo cuantitativo con un alcance exploratorio debido a que se enfocó a evaluar las competencias de los profesores que imparten la asignatura de Tecnología a nivel secundaria. Así, el estudio permitió recopilar información acerca de esta temática para determinar líneas de investigación especializada. Asimismo, fue no experimental porque no existió una manipulación deliberada de las variables al momento de aplicar el instrumento (Hernández, et al., 2010). Según Münch y Ángeles (2007), le correspondió un diseño transversal porque la recolección de los datos se llevó a cabo en un solo momento del tiempo (mayo de 2014). 


\section{Instrumento}

El instrumento que se elaboró fue un cuestionario (Tabla 1) que permitió recopilar la información e integrado por los apartados siguientes: 1) Datos generales de los participantes: Organizado en cinco reactivos que se refieren a obtener información básica de los profesores y 2) Competencias docentes de la asignatura de Tecnología: Organizado en los dominios de planeación, didáctica, evaluación y uso de TIC con un total de cuarenta ítems. El instrumento se elaboró con base en las aportaciones de Cardoso, et. al. (2013) Almerich, et. al. (2011) y Zabalza (2003). Los reactivos se evaluaron mediante una escala de frecuencia de cinco grados por lo que la escala utilizada correspondió a una de tipo Likert. Dicho cuestionario se estructuró con una escala Likert con cinco opciones de evaluación: 0 "No desarrollada"; 1 "Suficiente"; 2 "Regular"; 3 "Buena" y 4 "Excelente". Además, se calculó su confiabilidad, obteniendo las siguientes alfas de Cronbach: Planeación $=0.752$; Didáctica $=0.734$; Evaluación $=0.785$ y Uso de $T I C=0.795$. La alfa de Cronbach del instrumento fue de 0.761 lo cual significa que es confiable.

Tabla 1: Instrumento de investigación

\begin{tabular}{|c|c|c|c|c|c|}
\hline \multirow{2}{*}{$\frac{\text { Competencia Docente }}{\text { 1. Conocer el contenido del plan de estudios } 2011 \text { para la educación básica }}$} & 0 & 1 & 2 & 3 & 4 \\
\hline & & & & & \\
\hline 2. Identificar los 12 principios pedagógicos que sustentan el plan de estudios 2011 & & & & & \\
\hline 3. Diseñar planeación didáctica para la práctica docente & & & & & \\
\hline 4. Comprender propósitos de la asignatura de tecnología & & & & & \\
\hline 5. Formular estrategias didácticas con base en las características del grupo & & & & & \\
\hline 6. Comprender a quien alude el trabajo colaborativo y el propósito qué tiene & & & & & \\
\hline 7. Conocer el modelo basado en competencias & & & & & \\
\hline 8. Realizar evaluación de los aprendizajes & & & & & \\
\hline 9. Diferenciar la evaluación diagnóstica, formativa y sumativa & & & & & \\
\hline 10. Utilizar autoevaluación y coevaluación & & & & & \\
\hline 11. Diseñar rúbrica o matriz de verificación & & & & & \\
\hline $\begin{array}{l}\text { 12. Identificar instrumentos de evaluación para el aprendizaje que se apliquen en la asignatura } \\
\text { de tecnología }\end{array}$ & & & & & \\
\hline 13. Conocer competencias para la vida que se mencionan en el plan de estudios 2011 & & & & & \\
\hline 14. Identificar los campos de formación que se establecen en el mapa curricular & & & & & \\
\hline 15. Conocer el objeto de estudio de la asignatura de tecnología en la educación secundaria & & & & & \\
\hline 16. Conocer los saberes que se busca desarrollar en los alumnos en la asignatura de tecnología & & & & & \\
\hline 17. Desarrollar proyectos de tipo descriptivo y operativo & & & & & \\
\hline 18. Conocer la orientación de la asignatura de tecnología como espacio educativo & & & & & \\
\hline 19. Identificar las cuatro competencias específicas de la asignatura de tecnología & & & & & \\
\hline 20. Favorecer el enfoque pedagógico de la asignatura de tecnología & & & & & \\
\hline 21. Usar el análisis sistémico como método de trabajo de la asignatura de tecnología & & & & & \\
\hline 22. Utilizar proyectos como método de trabajo de la asignatura de tecnología & & & & & \\
\hline 23. Realizar lista de cotejo en la asignatura de tecnología & & & & & \\
\hline 24. Identificar aspectos a evaluar con la lista de cotejo & & & & & \\
\hline 25. Favorecer el papel del docente en la asignatura de tecnología & & & & & \\
\hline 26. Diseñar prácticas educativas relevantes en congruencia con el enfoque de la asignatura & & & & & \\
\hline 27. Identificar productos o evidencias que los alumnos elaboran en el laboratorio de tecnología & & & & & \\
\hline 28. Comunicarse en forma oral & & & & & \\
\hline 29. Comunicarse en forma escrita & & & & & \\
\hline 30. Comprender textos escritos & & & & & \\
\hline 31. Buscar información en Internet & & & & & \\
\hline 32. Evaluar aspectos relacionados a las capacidades de los alumnos en el laboratorio & & & & & \\
\hline 33. Conocer los ejes que estructuran los contenidos para el estudio del campo de la tecnología & & & & & \\
\hline 34. Utilizar las ocho estrategias didácticas propuestas para el estudio de la tecnología & & & & & \\
\hline 35. Establecer criterios para evaluar los contenidos & & & & & \\
\hline 36. Utilizar las TIC en el aula & & & & & \\
\hline 37. Diseñar ambientes de aprendizaje basados en TIC & & & & & \\
\hline 38. Emplear sistemáticamente Word, Excel y Power Point & & & & & \\
\hline 39. Utilizar internet & & & & & \\
\hline
\end{tabular}




\section{Muestra}

La muestra de la investigación se integró por 90 docentes que laboran en secundarias diurnas o generales (50 profesores) y secundarias técnicas (40 profesores) distribuidos en los campos de tecnologías de los alimentos, tecnología de la producción, tecnologías de la construcción, tecnologías de la información y comunicación así como tecnologías de la salud, los servicios y la recreación.

\section{RESULTADOS}

\section{Análisis factorial exploratorio}

La estimación de la validez de constructo del instrumento se llevó a cabo con base en un análisis factorial de componentes principales. La prueba estadística de KMO proporcionó un valor de 0.855 , por lo que se concluye una buena adecuación de los datos a un modelo factorial (Costello y Osborne, 2005). La prueba de esfericidad de Barlett fue estadísticamente significativo $\left(X^{2}=187.44, p=0.00\right)$. El análisis de validez de constructos fue: Para el componente de las competencias docentes relacionadas con la planeación se hallaron tres factores que explicaron el $72.85 \%$ de la varianza total; para el componente de didáctica, fueron cinco factores con el $75.88 \%$; para el componente de evaluación se identificaron tres con el $78.65 \%$ y, para el manejo de las TIC, se determinaron cinco factores con el $74.43 \%$ de la varianza total. Estos resultados son similares al estudio realizado por Mortis, et al. (2013), los cuales determinaron un valor de KMO igual a 0.952 , la prueba de esfericidad de Bartlett fue significativa $\left(X^{2}=119.44, p=0.00\right)$, así como extrajeron tres factores que explicaron el $70.1 \%$ de la varianza total de los puntajes.

\section{Principales resultados}

La muestra del estudio tuvo con respecto al género la siguiente distribución: $62 \%$ hombres y $38 \%$ mujeres. En cuanto a la modalidad de la secundaria fue un 55\% para generales y el $45 \%$ restante, técnicas. Mientras que la edad de los encuestados tuvo una media de 42.5 años con una desviación estándar de 9.4 años. Por su parte, el promedio de los años de servicio fue de 14.1 años con una desviación estándar de 6.8 años. Para la interpretación de las medias sobre las competencias docentes se establecieron tres niveles de consolidación de acuerdo al dominio diagnosticado: Nivel alto comprende valores superiores de 3 a 4; nivel medio comprende valores superiores de 2 hasta 3 y nivel bajo comprende valores superiores de 1 a 2 . Los resultados para cada componente se presentan en la tabla 1.

Tabla 2: Competencias docentes para la asignatura de tecnología

\begin{tabular}{|c|c|c|c|c|c|c|c|c|}
\hline \multirow{2}{*}{ Secundarias } & \multicolumn{2}{|c|}{ Planeación } & \multicolumn{2}{c|}{ Didáctica } & \multicolumn{2}{c|}{ Evaluación } & \multicolumn{2}{c|}{ Uso de TIC } \\
\cline { 2 - 9 } & Media & $\begin{array}{c}\text { Desviación } \\
\text { estándar }\end{array}$ & Media & $\begin{array}{c}\text { Desviación } \\
\text { estándar }\end{array}$ & Media & $\begin{array}{c}\text { Desviación } \\
\text { estándar }\end{array}$ & $\begin{array}{c}\text { Media } \\
\text { Desviación } \\
\text { estandar }\end{array}$ \\
\hline Generales & 2.77 & 0.21 & 2.85 & 0.61 & 2.79 & 0.49 & 2.81 & 0.42 \\
\hline Técnicas & 3.25 & 0.35 & 3.11 & 0.29 & 3.07 & 0.21 & 2.95 & 0.74 \\
\hline Total $(\mathrm{n}=90)$ & 3.01 & 0.28 & 2.98 & 0.45 & 2.93 & 0.35 & 2.88 & 0.58 \\
\hline
\end{tabular}

La distribución de las medias del estudio fueron: Para el primer componente, se halló que los docentes poseen un dominio alto; no obstante, los que pertenecen a la modalidad de secundarias generales tienen un dominio más bajo (medio) en comparación con los de la modalidad de secundarias técnicas. Para el segundo componente, se encontró que los encuestados tienen un dominio medio, destacando los que pertenecen a la secundarias técnicas con un dominio superior. Para el tercer componente, se obtuvo un dominio regular para los profesores; sin embargo, los de secundarias técnicas tuvieron un dominio alto con respecto a los de secundarias generales. Para el cuarto componente, se determinó un dominio regular y coincide para ambas modalidades escolares. En cuanto a las competencias docentes con nivel alto y medio se muestran en la tabla 3 y 4.

Con base en la tabla 3, para el componente de planeación los resultados fueron: Comprender propósitos de la asignatura de tecnología (3.34 \pm 0.18$)$; conocer el objeto de estudio de la asignatura de tecnología en la educación secundaria $(3.23 \pm 0.19)$ y conocer competencias para la vida que se mencionan en el plan de estudios 2011 para educación básica $(3.21 \pm 0.22)$. Para el componente de didáctica fueron: Utilizar proyectos como método de trabajo de la asignatura de tecnología ( $3.33 \pm 0.18)$; comunicarse en forma oral $(3.30 \pm 0.19)$ y comunicarse en forma escrita $(3.27 \pm 0.21)$. Para el componente de evaluación fueron: Identificar productos o evidencias que los alumnos elaboran para su evaluación en el laboratorio de tecnología (3.21 \pm 0.25$)$; realizar evaluación de los aprendizajes $(3.14 \pm 0.29)$ y diferenciar la evaluación diagnóstica, formativa y sumativa $(3.11 \pm 0.31)$. Para el componente de uso de TIC solamente existió una que fue la de buscar información en Internet (3.17 \pm 0.28$)$. 
Tabla 3: Competencias con nivel alto (continúa)

\begin{tabular}{|c|c|}
\hline Componente & Media \\
\hline \multicolumn{2}{|l|}{ Planeación } \\
\hline Comprender los propósitos de la asignatura de tecnología & 3.34 \\
\hline Conocer el objeto de estudio de la asignatura de tecnología en la educación secundaria & 3.23 \\
\hline $\begin{array}{l}\text { Conocer competencias para la vida que se mencionan en el plan de estudios } 2011 \text { para educación } \\
\text { básica }\end{array}$ & 3.21 \\
\hline Conocer los saberes que se busca desarrollar en los alumnos en la asignatura de tecnología & 3.19 \\
\hline Diseñar planeación didáctica para la práctica docente & 3.10 \\
\hline Conocer la orientación de la asignatura de tecnología como espacio educativo & 3.06 \\
\hline \multicolumn{2}{|l|}{ Didáctica } \\
\hline Utilizar proyectos como método de trabajo de la asignatura de tecnología & 3.33 \\
\hline Comunicarse en forma oral & 3.30 \\
\hline Comunicarse en forma escrita & 3.27 \\
\hline Comprender textos escritos & 3.09 \\
\hline Favorecer el papel del docente en la asignatura de tecnología & 3.04 \\
\hline Formular estrategias didácticas con base en las características del grupo & 3.01 \\
\hline \multicolumn{2}{|l|}{ Evaluación } \\
\hline $\begin{array}{l}\text { Identificar productos o evidencias que los alumnos elaboran para su evaluación en el laboratorio } \\
\text { de tecnología }\end{array}$ & 3.21 \\
\hline Realizar evaluación de los aprendizajes & 3.14 \\
\hline Diferenciar la evaluación diagnóstica, formativa y sumativa & 3.11 \\
\hline Evaluar aspectos relacionados a las capacidades de los alumnos en el laboratorio de tecnología & 3.09 \\
\hline $\begin{array}{l}\text { Identificar instrumentos de evaluación para él aprendizaje que se apliquen en la asignatura de } \\
\text { tecnología }\end{array}$ & 3.06 \\
\hline \multicolumn{2}{|l|}{ Manejo de TIC } \\
\hline Buscar información en Internet & 3.17 \\
\hline
\end{tabular}

Tabla 4: Competencias con nivel medio

Componente Planeación

Identificar los campos de formación de la educación básica que se establecen en el mapa curricular Conocer los ejes que estructuran los contenidos para el estudio del campo de la tecnología Identificar los 12 principios pedagógicos, que sustentan el plan de estudios 2011 para educación básica Didáctica

Conocer el modelo basado en competencias

Favorecer el enfoque pedagógico de la asignatura de tecnología

Comprender a quien alude el trabajo colaborativo y el propósito qué tiene

Desarrollar proyectos para la articulación de acciones y conocimientos de tipo descriptivo y operativo

Usar el análisis sistémico como método de trabajo de la asignatura de tecnología

Utilizar las ocho estrategias didácticas propuestas para el estudio de la tecnología Evaluación

Establecer criterios para evaluar los contenidos conceptuales, procedimentales y actitudinales

Identificar aspectos a evaluar con la lista de cotejo

Utilizar autoevaluación y coevaluación

Realizar lista de cotejo en la asignatura de tecnología

Diseñar rúbrica o matriz de verificación Manejo de TIC

Utilizar las TIC en el aula

Utilizar internet

Diseñar ambientes de aprendizaje basados en TIC

Emplear sistemáticamente Word, Excel y Power Point

A partir de la tabla 4, se halló que para el componente de planeación, la competencia de identificar los campos de formación de la educación básica $(2.80 \pm 0.34)$; para el componente de didáctica fue la de conocer el modelo basado en competencias $(2.98 \pm 0.36)$; para el de evaluación fue la de establecer criterios $(2.84 \pm 0.44)$ y para el manejo de TIC fue la de utilizarlas en el aula $(2.93 \pm 0.51)$. Cabe aclarar que en el estudio, no se obtuvo una media menor a 2 por lo que no existen resultados acerca del nivel de dominio bajo de las competencias docentes para los profesores que imparten la asignatura de tecnología. Por último, se llevaron a cabo comparaciones entre las variables de modalidad así como la edad con la finalidad de identificar la existencia de diferencias en términos estadísticos: Se encontraron diferencias significativas en planeación $(t=-8.051, p=0.01<0.05)$ y didáctica $(t=-2.478, p=0.01<0.05)$, entre la 
modalidad de secundaria general y la modalidad técnica, con un $95 \%$ de confianza. Este resultado permite deducir que los encuestados presentan un dominio distinto en sus competencias. En tanto, para los factores de evaluación y uso de TIC no existió una diferencia. Por lo que los profesores perciben en forma igual las competencias que poseen en estos componentes.

Se encontraron diferencias significativas en el empleo de las TIC, según la edad del profesorado con un $95 \%$ de confianza (ANOVA, $F=26.764, p=0.01<0.05$ ). Este resultado hace referencia a la existencia de diferentes niveles de dominio en dichas competencias, en donde se distinguió que los profesores de menor edad poseen una consolidación en estas herramientas tecnológicas en contraste con lo de mayor edad. En lo que respecta a este componente, es posible inferir que el rechazo y la falta de utilización de las TIC en los docentes es una realidad, que no facilita la incorporación de innovaciones al proceso de enseñanzaaprendizaje, para generar interés y participación en los alumnos en la realización de las actividades formativas, por lo que se confirma lo hallado por Rangel y Peñalosa (2013) y Vera, et. al., (2014). Del mismo modo, los profesores solamente poseen competencias de uso ofimático por lo que también se presenta el mismo resultado hallado por Peinado, et. al. (2011) y Almerich, et. al. (2011).

Finalmente, realizando una comparación de los resultados de este estudio evaluativo con otros, se presenta una situación similar en cuanto al nivel de consolidación medio de las competencias docentes en las áreas de planeación de estrategias didácticas e instrumentos de evaluación (Cardoso, et al., 2013). Del mismo modo, Hodgson, et al., (2014) encontró en su investigación sobre las competencias científicas de los profesores, la existencia de niveles regulares sobre la asesoría de tesis, trabajo colaborativo entre el investigador con sus alumnos y la participación en la escritura de textos académicos.

\section{CONCLUSIONES}

Los resultados encontrados con este trabajo de investigación permiten obtener las siguientes conclusiones: 1) Se logró el objetivo general porque se evaluó el nivel de dominio de las competencias docentes en los factores de planeación, didáctica, evaluación y uso de TIC de los profesores de educación secundaria de la asignatura de tecnología; 2) Los profesores se autoperciben con un nivel alto de dominio en las competencias relacionadas con la planeación, mientras que para los otros tres componentes (didáctica, evaluación y manejo de TIC), los encuestados tienen un dominio regular; 3) Los hallazgos corresponden a los docentes que imparten la asignatura de tecnología en donde las investigaciones en México son escasas; 4) Se recomienda a las IES establecer cursos de capacitación y de formación continua para estos docentes con la finalidad de brindarles estrategias que les permitan consolidar sus competencias académicas y, 5) Se sugiere que se realicen otros estudios que permitan corroborar, ampliar o refutar los resultados obtenidos para los estados que conforman a México, así como en las secundarias de régimen privado.

\section{REFERENCIAS}

Alcira, A., Escalante, M. y León, A. La educación en tecnología: Un reto para la educación básica venezolana. Educere, ISSN: 1316-4910 (en línea), 12(43), 731-740, 2008, http://sociales.redalyc.org/articulo.oa?id=35614570009. Acceso: 15 de enero (2014)

Almerich, G., Suárez - Rodríguez, J., Belloch, C. y Bo, R. Las necesidades formativas del profesorado en TIC: perfiles formativos y elementos de complejidad. Relieve, 17(2), 1-28 (2011)

Bozu, Z. y Canto, P. El profesorado universitario en la sociedad del conocimiento: competencias profesionales docentes. Revista de Formación e Innovación Educativa Universitaria, ISSN: 1989 - 0257, 2 (2), 87 - 97, (2009)

Cardoso, E., Cerecedo, M. y Vanegas, E. (2013). Las competencias docentes en los programas de posgrado en administración: Un estudio diagnóstico, doi: 10.4067/S0718-50062013000200006, Formación Universitaria (en línea), 6(2), pp. 43 - 50, http://www.scielo.cl/scielo.php?script=sci_pdf\&pid=S0718$50062013000200006 \& \operatorname{lng}=e s \& n r m=i s o \&$ tIng $=e s$.

Costello, A. y Osborne, J. Best practices in exploratory factor analysis: four recommendations for getting the most from your analysis. Practical Assessment, Research and Evaluation, 10(7), 1 - 9, (2005)

Diario Oficial de la Federación. Acuerdo 593. México: Diario Oficial de la Federación, 2011, http://dof.gob.mx/nota_detalle.php?codigo=5205626\&fecha=22/08/2011. Acceso: 16 de enero (2014)

Donoso, S. El perfeccionamiento docente en Chile (1990 - 2007): ¿Estado versus mercado?. Revista Brasileira de Educacao, 13(39), 437 - 454, (2008)

Ferreras, M. Aportes para la implementación de la educación de la tecnología. Buenos Aires: Instituto Nacional de Educación Tecnológica, (2004) 
Gibson, M. Assessing Teaching Skills with a Mobile Simulation, doi: 10.1080/21532974.2013.10784720, Journal of Digital Learning in Teacher Education (en línea), 30(1), 4 - 10, (2013), http://www.tandfonline.com/doi/pdf/10.1080/21532974.2013.10784720

Hernández, R., Fernández, C. y Baptista, P. Metodología de la investigación. México: Mc Graw Hill, 2010.

Hodgson, Y., Varsavsky, C. y Matthews, E. Assessment and teaching of science skills: whole of programme perceptions of graduating students, doi: 10.1080/02602938.2013.842539, Assessment and Evaluation in Higher Education (en línea), 39(5), 515 - 530, 2014,

http://www.tandfonline.com/doi/full/10.1080/02602938.2013.842539

Mortis, S., Valdés, Á., Ángulo, J., García, R. y Cuevas, O. Competencias digitales en docentes de educación secundaria. Municipio de un Estado del Noroeste de México. Perspectiva Educacional, formación de profesores, ISSN: 0716-0488 (en línea), 52(2), 135 - 153, 2013, http://www.redalyc.org/articulo.oa?id=333328170007. Acceso: 5 de diciembre (2014)

Münch, L. y Ángeles, E. Métodos y técnicas de investigación, México: Trillas, (2007)

Namakforoosh, J. M. Metodología de la investigación. México: Limusa, (2011)

Palominos, F., Mendez, M. y Barrera, R. Sistema de Perfeccionamiento Orientado a Competencias para Docentes de la Educación Superior, doi: 10.4067/S0718-50062014000300003, Formación Universitaria (en línea), 7(3), 11-22, 2014, http://www.scielo.cl/pdf/formuniv/v7n3/art03.pdf

Pantoja, A. y Huertas, A. Integración de las TIC en la asignatura de tecnología de educación secundaria. Revista Pixel - Bit. Revista de Medios y Educación, ISSN: 1133 - 8482 (en línea), 37, 225 - 337, 2010, http://www.redalyc.org/articulo.oa?id=36815118018. Acceso: 17 de enero (2014)

Peinado, S., Bolívar, J. y Briceño, L. Actitud hacia el uso de la computadora en docentes de educación secundaria. Revista Universitaria Arbitrada de Investigación y Diálogo Académico, ISSN: 1690 - 7760 (en línea), 7(1), 86 - 105, 2011, http://conhisremi.iuttol.edu.ve/pdf/ARTI000115.pdf. Acceso: 20 de enero (2014)

Perrenoud, P. Diez nuevas competencias para enseñar. México: Quebecor World, (2004)

Poggi, M. Innovaciones educativas y escuelas en contextos de pobreza. Evidencias para las políticas de algunas experiencias en América Latina. Buenos Aires: Instituto Internacional de Planeamiento de la Educación (IIPE) - UNESCO, (2011)

Ramírez, A. y Escalante, M. Educación en tecnología: un aporte desde Fe y Alegría. Caracas: Fe y Alegría (Colección Procesos Educativos No. 27), (2007)

Rodríguez, G. Ciencia, Tecnología, Sociedad: una mirada desde la educación en Tecnología. Revista Iberoamericana de Educación, ISSN: 1681 - 5663 (en línea), 18, 107 - 143, 1998, http://www.rieoei.org/oeivirt/rie18a05.pdf. Acceso: 15 de enero (2014)

Rangel, A. y Peñalosa, E. Alfabetización digital en docentes de Educación Superior: construcción y prueba empírica de un instrumento de evaluación. Revista Pixel - Bit. Revista de Medios y Educación, ISSN: 1133 - 8482 (en línea), 43, 9 - 23, 2013, http://www.redalyc.org/articulo.oa?id=36828074002. Acceso: 15 de enero (2014)

SEP. Antología. México: Secretaría de Educación Pública, (2009)

SEP. Plan de Estudios 2011. Educación Básica. México: Secretaría de Educación Pública, (2011)

Tejada, J. Competencias docentes. Profesorado. Revista de Currículum y Formación de Profesorado, 13(2), $1-15,(2009)$

Tobon, S. Aspectos básicos de la formación basada en competencias. Talca: Proyecto Mesesup, (2006).

Valdés, Á., Vera, J. y Carlos, E. Competencias científicas en estudiantes de posgrado de ciencias naturales e ingenierías. Sinéctica, ISSN: 1665 - 109X (en línea), 39, 1-17, (2012)

http://www.redalyc.org/articulo.oa?id=99826889012. Acceso: 15 de enero (2014)

Vera, J., Torres, L. y Martínez, E. Evaluación de competencias básicas en TIC en docentes de educación superior en México. Pixel - Bit. Revista de Medios Educación, ISSN: 1133 - 8482 (en línea), 44, 143 - 155, 2014, http://www.redalyc.org/articulo.oa?id=36829340010. Acceso: 15 de enero (2014)

Zabala, A. y Arnau, L. La enseñanza de las competencias. Aula de Innovación Educativa, 161, 40 - 46, (2007)

Zabalza, M. A. Competencias docentes del profesorado universitario. Calidad y desarrollo profesional. Madrid: Narcea, (2003) 